\title{
Quality Improvement in Ambulatory Surgery Centers: A Major National Effort Aimed at Reducing Infections and Other Surgical Complications
}

\author{
Kristina K. Davis ${ }^{\mathrm{a}}$, Vrinda Mahishi ${ }^{\mathrm{a}}$, Robbie Singal ${ }^{\mathrm{b}}$, Richard D. Urman ${ }^{\mathrm{c}, \mathrm{e}}$, \\ Melissa A. Miller ${ }^{d}$, Marcia Cooke ${ }^{a}$, William R. Berry ${ }^{b}$
}

\begin{abstract}
Background: Surgical volume has shifted significantly from inpatient to outpatient settings, including free-standing ambulatory surgery centers (ASCs). Approaches to quality improvement (QI) and surveillance used in hospitals are not always appropriate to the ambulatory setting.

Methods: We recruited 665 ASCs in 47 US states to participate in an intervention to improve safe practice through implementation of a surgical safety checklist and infection control practices. Areas for partner contribution included recruitment, project development, content development and delivery, clinical subject matter expertise, data analysis, and facility coaching.
\end{abstract}

Results: Barriers to implementation and data collection were encountered during the project, requiring revisions to the implementation plan. Project activities, such as facility recruitment, data measurement, and implementation strategies were modified to meet ASCspecific needs. Several ASC-specific tools were designed.

Conclusions: The increasing number of patients being cared for in ASCs makes it essential to better understand how to implement quality improvement projects in that environment. Tailoring interventions to the ASC's unique needs is necessary.

Keywords: Checklist; Infection control; Quality improvement; Ambulatory surgery center; Safety; Implementation

Manuscript submitted September 4, 2018, accepted October 15, 2018

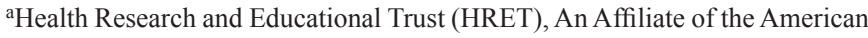
Hospital Association, Chicago, IL, USA

'Ariadne Labs, A Joint Center Between Brigham and Women's Hospital and the Harvard T.H. Chan School of Public Health, Boston, MA, USA

${ }^{c}$ Department of Anesthesiology, Perioperative and Pain Medicine, Harvard Medical School, Brigham and Women's Hospital, Boston, MA, USA

${ }^{\mathrm{d}}$ Division of Healthcare-Associated Infections, Center for Quality Improvement and Patient Safety, Agency for Healthcare Research and Quality (AHRQ), Rockville, MD, USA

${ }^{\text {e}}$ Corresponding Author: Richard D Urman, Department of Anesthesiology, Perioperative and Pain Medicine, Brigham and Women's Hospital, Harvard Medical School, Boston, MA 02115, USA. Email: rurman@bwh.harvard.edu

doi: https://doi.org/10.14740/jocmr3603w

\section{Introduction}

In the United States, more than 25 million surgeries a year are performed in over 5,300 ambulatory surgery centers (ASCs) [1]. Cases performed in ASCs due to advances in surgical technology and anesthesia led to convenience for patients and providers as well as cost savings [2-4]. Ambulatory care accounts for the majority of surgical care in the United States [4]. ASCs have begun to offer a wider range of procedures, and the number of higher-risk patients operated on in ASCs continues to grow $[2,5]$. As the trend for outpatient surgery continues to shift from the inpatient to the ASC setting, continued attentions to quality and infection control are priority areas for ASC leaders focused on delivering high quality care [3].

In 2011, the Agency for Healthcare Research and Quality (AHRQ) requested proposals for development and demonstrations of a Surgical Unit-based Safety Program (SUSP) to reduce surgical site infections (SSI) and other surgical complications program [6]. In response to the task order, an initial team was assembled from four diverse national organizations. The project team aimed to increase surgical safety through the adaptation of the Comprehensive Unit-based Safety Program (CUSP) framework and implementation of the World Health Organization's (WHO) Safe Surgery Checklist and other evidence-based infection prevention practices. Based in part on the Institute for Healthcare Improvement (IHI) Breakthrough Collaborative Model, the group developed a multifaceted safe-surgery intervention focused on both technical and cultural components, an approach that was previously successful in reducing central line and urinary tract infections in inpatient settings [7, 8].

Because of the large number of procedures performed in ASCs, AHRQ expanded the focus of its safe surgery initiatives to include this setting. At the time, it was estimated that more than 7 million people worldwide were negatively impacted by surgical complications yearly in all health care settings; however, the rates of these complications in ASCs were unknown [9]. What was known came from outbreak investigations or small-scale pilot studies [10]. AHRQ funded the first project to focus on the national implementation of a quality improvement (QI) intervention within the ASC setting with the primary goal of reducing SSIs and other major surgical complications. This paper details implementation of a large scale QI intervention in the ASC environment and how the project developed and 
Table 1. Partner Organizations of the National Project Team

\author{
Partner \\ Harvard School of Public Health (HSPH) \\ Ambulatory Surgery Center Association (ASCA) \\ ASC Quality Collaboration (ASC QC) \\ Westat \\ South Carolina Hospital Association (SCHA) \\ Institute for Healthcare Improvement (IHI)
}

\title{
Extended faculty network
}

American Academy of Orthopaedic Surgeons (AAOS)

American Association of Nurse Anesthetists (AANA)

Society for Ambulatory Anesthesiology (SAMBA)

Association of periOperative Registered Nurses (AORN)

Association for Professionals in Infection Control and Epidemiology, Inc. (APIC)

International Association of Healthcare Central Service Material Management (IAHCSMM)

Accreditation Association for Ambulatory Health Care (AAAHC)

The Joint Commission (TJC)

American Society for Gastrointestinal Endoscopy (ASGE)

Association for the Advancement of Medical Instruments (AAMI)

Representatives from state ambulatory surgery associations and ASC management companies

adapted in hopes that others carrying out similar projects may be able to learn from this project and implement successful and sustainable interventions.

\section{Materials and Methods}

\section{National project team}

Successful QI projects generally require strong partnerships. While the key partners of the national project team (NPT) had a large breadth of experience in QI and implementing safe surgery interventions, working in the ASC setting was new for most in- volved. This gap was filled by engaging additional partners from organizations with experience working in ASCs. The strengths of each organization were leveraged to create a robust NPT (Table 1). Areas for partner contribution included: recruitment, project development, content development and delivery, clinical subject matter expertise, data analysis and facility coaching.

\section{Overview of project}

The project was implemented in eight cohorts over the course of 4 years (Table 2). The project was submitted to our institution's IRB for approval. As quality improvement, this project was granted exemption by the IRB. Variations between cohorts,

Table 2. Cohort Participation: The Project Was Implemented in Eight Cohorts Over the Course of 4 Years

\begin{tabular}{lll}
\hline Cohort & \# of facilities & Participation dates \\
\hline Cohort 1 & 53 & April, 2013 - April, 2014 \\
Cohort 2 & 109 & September, 2013 - September, 2014 \\
Cohort 3 & 69 & April, 2014 - May, 2015 \\
AAOS specialty Cohort & 12 & September, 2014 - September, 2015 \\
Cohort 4 & 100 & September, 2014 - October, 2015 \\
Cohort 5 & 103 & March, 2015 - April, 2016 \\
Cohort 6 (endoscopy-only) & 119 & July, 2015 - April, 2016 \\
Cohort 7 & 82 & September, 2015 - July, 2016 \\
\hline
\end{tabular}

AAOS: American Academy of Orthopaedic Surgeons. 


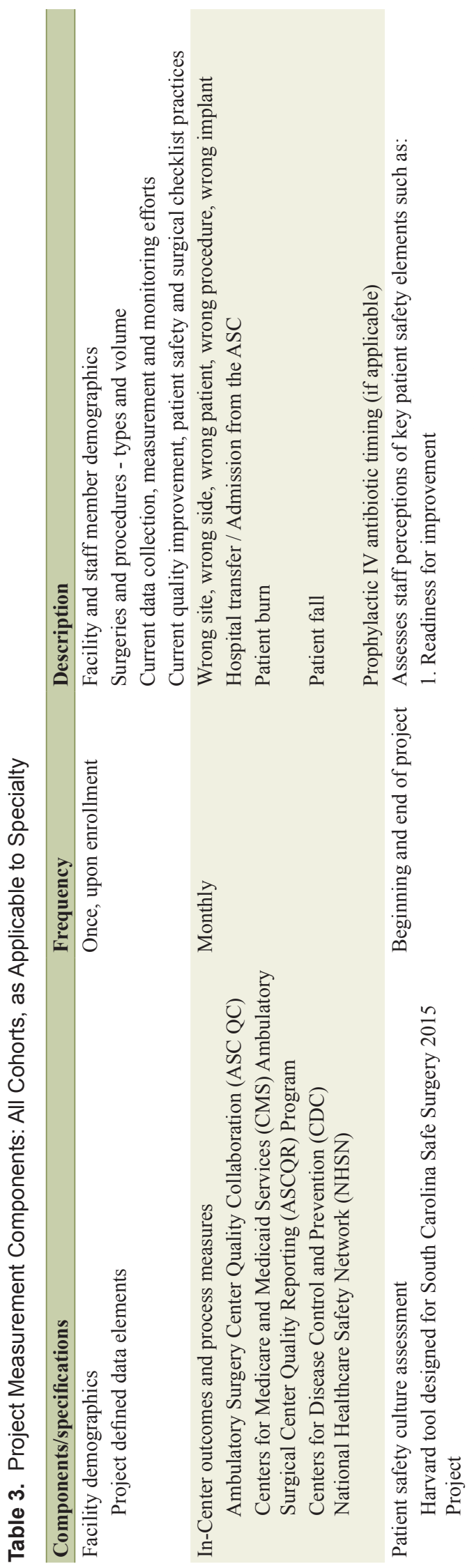

including how the model grew and developed, are detailed below. In general, the program was designed as a 12-month project for each cohort. When ASCs joined the project, they committed to the following activities: submission of baseline and monthly process and outcome data, completion of baseline and follow-up safety culture assessments, assessing patient satisfaction (Table 3), attending scheduled educational events (Table 4), working as a team to discuss progress and improve performance, and providing feedback on program elements. A secure, web-based data-collection tool was maintained for participants. Participants also used its reporting functions to view results and aggregate rates. Both quantitative and qualitative analyses were conducted by the NPT. This paper focuses on the design of a quality improvement intervention.

\section{Initial implementation plan}

The first cohort was launched in April 2013 (Fig. 1). The ASC program was structured initially with consortium leads (CLs) who functioned as intermediaries between the national project and ASCs. A majority of the CLs came from state ambulatory surgery center associations and/or ASC management companies. The CLs were administrators or nurses who had not been uniformly trained in quality improvement. Once the CL committed, the CL was responsible for recruiting and enrolling a minimum of 10 ASCs in their state or management company. This approach was modeled after recruitment strategies previously used for successful inpatient QI projects [7, 11, 12].

Under the consortium model, the CL was trained and supported all phases of recruitment, implementation and sustainability, serving as a liaison between the ASC and the NPT. Facility leads and staff from the ASCs were expected to participate in the educational content and data-collection activities of the project; but CLs were responsible for ensuring that participation and data-submission rates among ASCs were over $70 \%$. The project, however, was voluntary and no incentives were provided directly to the ASCs.

\section{Implementation plan for later cohorts}

Starting with Cohort 3 , the project redirected towards specialty-based cohorts using the lessons learned from previous cohorts. Cohort 3 was the first cohort focused on incision-based surgery in freestanding ASCs (excluding hospital outpatient departments (HOPDs)) where CLs were not used. Given this change in design, recruitment was also modified. Cohorts were organized based on ASC type as opposed to state or management company. Furthermore, partners continued to assist with recruitment while recruitment webinars were held by ASCA and state ambulatory surgery center associations. However, the NPT also expanded recruitment efforts to direct marketing at national and regional conferences and with other efforts. If the leadership of an ASC was interested, a representative (a facility lead) often enrolled directly on the project website. Each ASC was then contacted by NPT staff to confirm enrollment and discuss the project activities before launch of the cohort.

Under this new model, the role of the CL was eliminated; 
Table 4. Educational Program*

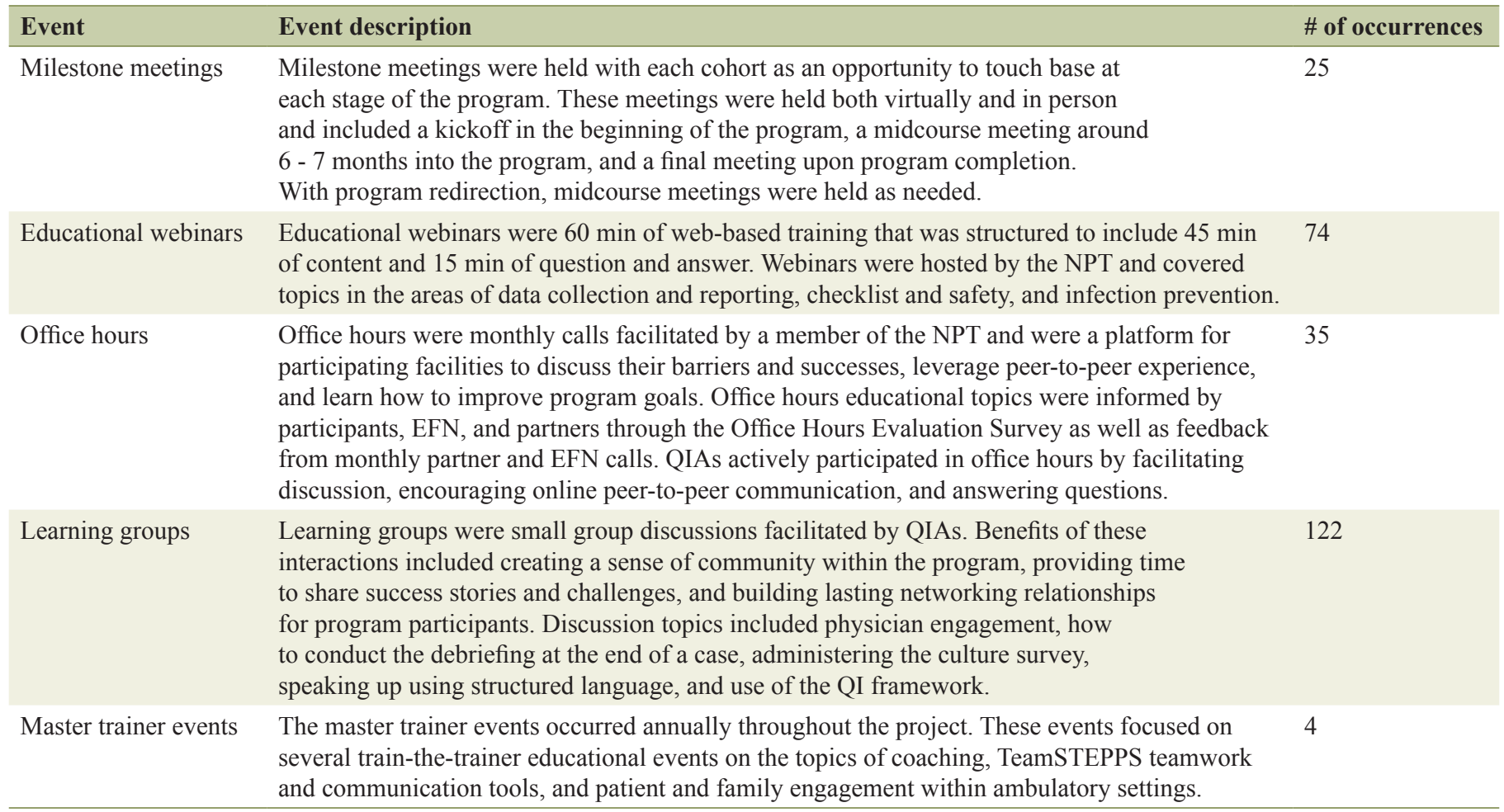

*Number of meetings between April 24, 2013 and September 29, 2016. NPT: national project team; EFN: extended faculty network; QIA: quality improvement advisors; QI: quality improvement.

quality improvement advisors (QIAs) were hired to fill this gap by working directly with the facility leads at each ASC. The QIAs were health care professionals trained in coaching and quality improvement. One was a nurse with an extensive coaching background in clinical and non-clinical settings and the other QIA was a former commercial airline pilot with ex-

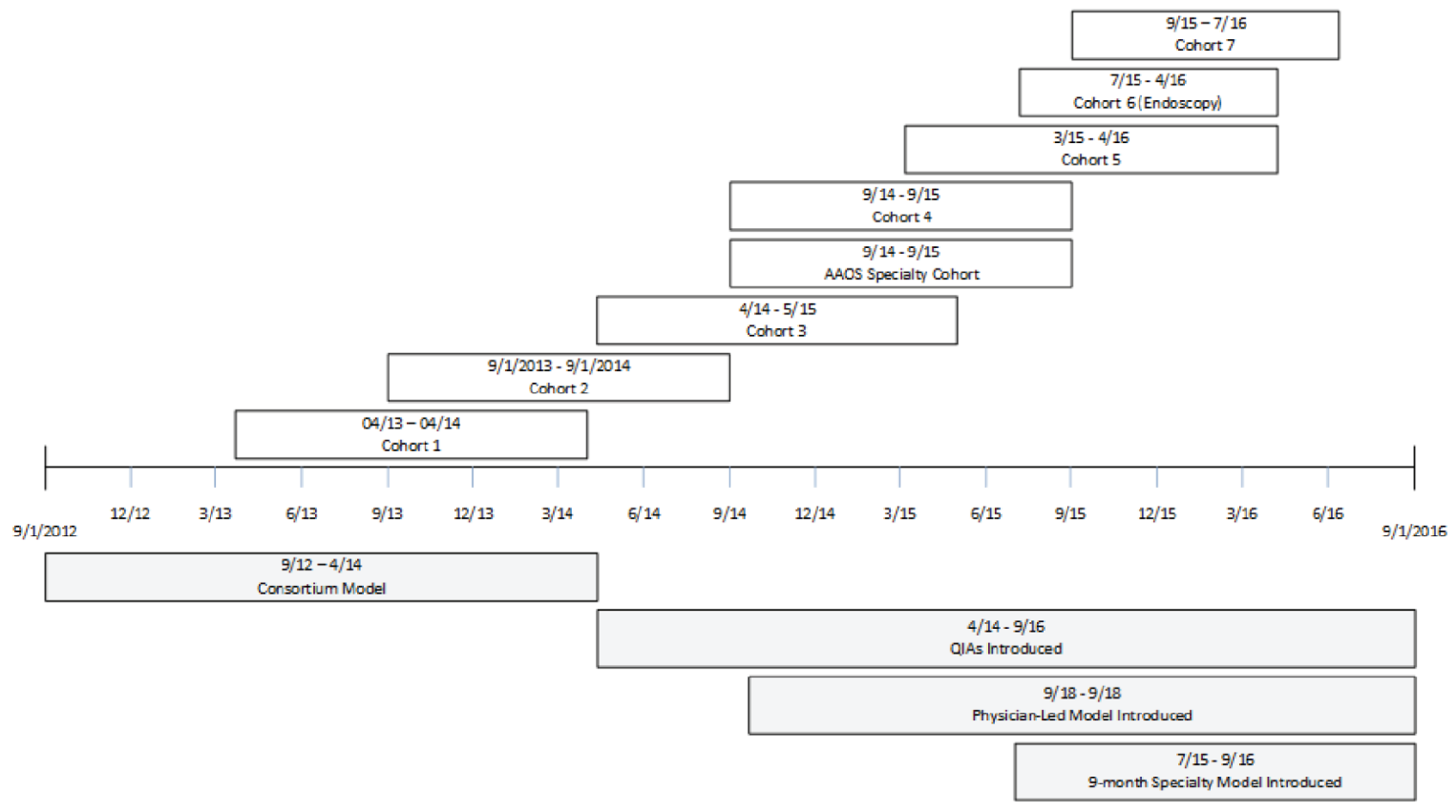

Figure 1. Timeline of events. 
Table 5. Unexpected Events: Cohorts 3 - 7, AAOS Pilot

\begin{tabular}{|c|c|c|}
\hline Components/specification & Frequency & Description \\
\hline Unexpected events & $\begin{array}{l}\text { As they occur, } \\
\text { C3 and beyond }\end{array}$ & $\begin{array}{l}\text { For each event listed below, facilities provide additional information including the procedure } \\
\text { that took place in the ASC, date of the event, date of the procedure, reason for transfer/ } \\
\text { admission, and how the facility found out about the event: } \\
\text { 1. Wrong side, site, patient, procedure, implant } \\
\text { 2. Hospital transfer/admission from the ASC } \\
\text { 3. Hospitalization or ED visit within } 48 \text { h of discharge from ASC } \\
\text { 4. Reoperation within } 48 \text { h of discharge from ASC } \\
\text { 5. SSI } \\
\text { 6. Other infection (Non-SSI) } \\
\text { 7. Cancelled procedure } \\
\text { 8. Other unexpected event } \\
\text { In addition, cohort specific events include: } \\
\text { 1. Wrong side, site anesthesia block (AAOS cohort) } \\
\text { 2. Unplanned intervention- resolved in the ASC (endoscopy cohort) }\end{array}$ \\
\hline
\end{tabular}

ASC: ambulatory surgery center; ED: emergency department; SSI: surgical site infection; AAOS: the American Academy of Orthopaedic Surgeons

perience using checklists and a background in QI in a hospital setting. The QIAs interacted with facility leads to reinforce webinar messages and initiate tailored coaching plans. Given the large number and geographic dispersion of facilities participating, the QIAs worked remotely via learning groups of five to six ASCs and one-on-one coaching calls to provide additional implementation support throughout the project.

The NPT convened an extended faculty network (EFN) to provide support to address identified programmatic gaps. The EFN consisted of subject matter experts from professional societies, accrediting bodies, state associations and management companies (Table 1). The NPT engaged the EFN in delivering webinar content, facilitating office hours, participating on a listserv, and providing essential expertise on learning groups.

Beginning in Cohort 3, data requirements were modified, and facilities were asked to provide additional details on unexpected events that may have an impact on patient safety and care that were not represented by the outcome measures collected (Table 5). Additionally, the validated the ASC Survey on Patient Safety (ASC-SOPS) was implemented as a culture assessment beginning with this cohort [13].

\section{Specialty cohorts}

Two additional specialty-specific models were tested. The American Academy of Orthopaedic Surgeons (AAOS) Specialty Cohort was designed as a 12-facility cohort in partnership with AAOS. The model focused on implementation emphasizing the importance of strong physician leadership support to achieve QI success. The facilities were recruited through the AAOS interaction with interested orthopedic surgeons.

The second specialty cohort focused on endoscopy centers (Cohort 6). Outcome measures and unexpected events were modified to better reflect this non-incision-based environment. This cohort used an abbreviated 9-month timeline, with additional content focused on scope reprocessing and the recently adapted surgical safety checklist for endoscopy procedures incorporated into the curriculum and irrelevant material removed. The unexpected events for specialty cohorts were modified to better capture specialty-specific events.

\section{Results}

Partnerships with the state ambulatory surgery center associations and management companies were invaluable in recruiting facilities for the first two cohorts, but it became increasingly difficult to recruit ASCs for additional cohorts under this model.

Requiring the CL to recruit facilities and support implementation inhibited the NPT from recruiting ASCs outside of states with active state ambulatory surgery center associations and decreased the ability to reach across all 50 states, DC, and Puerto Rico. It was not possible to recruit ASCs that were unaffiliated with their state's ambulatory surgery center association, and some states have fewer than 10 ASCs. This model depended on the QI skills and experience level of the CL, with some CLs able to take a more active role in encouraging data submission and participation than others, resulting in variable and inconsistent experiences at participating ASCs.

Cohorts 1 and 2 were open to all ASCs, HOPDs, and endoscopy centers within the participating states/management companies. However, this generic approach to content delivery was ineffective for participating ASCs. Educational material required adjustment based on its relevance to specific procedure types (e.g., endoscopy, ophthalmology, pain management). In addition, the NPT learned that there are differences between ASCs and HOPDs, which more closely resemble inpatient ORs than they do freestanding ASCs.

The content offered and delivery method used in Cohorts 1 and 2 did not work well within the ASC setting. For instance, scheduling in-person collaborative meetings was difficult be- 
Table 6. Number of Unexpected Events Reported: Cohorts 3 - 7, AAOS Pilot

\begin{tabular}{lll}
\hline Event description & Number of events reported & Percent of all events reported \\
\hline Same day cancelled procedure (Cohort 6 only) & 2,602 & $54 \%$ \\
Other & 748 & $16 \%$ \\
Hospital transfer/admission from the ASC & 552 & $12 \%$ \\
Hospital admission/ED visit within 48 h of discharge from the ASC & 479 & $10 \%$ \\
Surgical site infection & 143 & $3 \%$ \\
Unplanned intervention resolved in the ASC & 92 & $2 \%$ \\
Cancelled surgery due to medical reason (Cohort 7 only) & 104 & $2 \%$ \\
Reoperation within 48 h of discharge from the ASC & 57 & $1 \%$ \\
Other infection (Non-SSI) & 17 & $0 \%$ \\
\hline
\end{tabular}

The highest number of events was related to same day cancellations, hospital transfer/admission, and hospital/ED visit within $48 \mathrm{~h}$, and "other". The most common reasons for hospital transfer/admission from the ASC were unstable vital signs, airway management concerns, and cardiac issues. The most common reasons for "other" were unexpected preoperative findings such as high blood pressure, fever, high glucose and patients unprepared for procedure.

cause ASC staff did not have dedicated time or resources set aside for this project to travel to meetings. Some ASCs in Cohorts 1 and 2 also reported a lack of time and resources to work on this project.

The rates of ASC QC outcome measures among participating Cohort 1 and 2 facilities were extremely low, with an average of $0.03 \%$ each for wrong site/wrong side/wrong patient/wrong procedure/wrong implant, patient fall, and patient burn and $0.06 \%$ for hospital transfer/admission. These very low complication rates made the detection of further decreases statistically impossible because of the limitation in the number of outcomes being examined. The addition of unexpected events data collection provided information about the types and frequency of other events that occur in the ASCs extending beyond the data elements more routinely collected for quality and patient safety monitoring (Table 6). The highest number of events were related to hospital transfer/admission or hospital/ ED visit within $48 \mathrm{~h}$.

Challenges occurred using the surgeon-led implementation model as maintaining high levels of engagement was difficult. Both high facility attrition and low participation rates were observed, with four out of 12 facilities disenrolling early in the cohort and a maximum of six facilities submitting data in any given month.

Compared to other cohorts, data-submission rates and engagement were higher in the endoscopy cohort. Seventy-six of the 120 enrolled facilities remained actively engaged throughout the course of the cohort. Many representatives from facilities reported that limited endoscopy-specific patient-safety programs were available. As a result, they were eager to participate in a large, national learning project.

\section{Discussion}

This project was the first major national effort aimed at reducing infections and other surgical complications in the ASC setting. Using the same rapid cycle improvement strategy facilities were encouraged to employ the NPT tested multiple approaches to intervention delivery: the CL model, the QIA model, physician-led model, and single-specialty, 9-month cohort model. While not all approaches used were successful, there were lessons learned from each approach that may benefit others who implement programs in this environment.

From information collected throughout the project, the NPT learned about specific barriers which informed development of each of the implementation models trialed. Some facilities reported challenges related to a lack of staff, time and resources for this project. ASC staff often may not have formal training or experience in QI work [14]. Therefore, the project was modified to accommodate the need for more implementation support through coaching, with the addition of QIAs who provided a more structured approach to QI. Maintaining facility engagement was a challenge throughout the project. Participation in the project was entirely voluntary, and incentives for participation were not available. Occasionally, facility issues including staff turnover presented challenges. The main predictors of engagement and successful implementation at the facility level were strong leadership support from administrative and clinical leads, dedicated time and resources to devote to project activities, and committed physician quality champions.

Existing education was augmented with more robust, ASC-specific components. The new approach allowed the NPT, with feedback from facilities, to design educational materials to address the specific needs of the cohort's specialty. Additional content on project spread, sustainability, disinfection and sterilization techniques, and TeamSTEPPSTM (Team Strategies and Tools to Enhance Performance and Patient Safety) tools were added based on identified priority areas. In addition, videos and modules were developed specifically for ASCs. In-person meetings were discontinued, and content became entirely web-based. All webinars were recorded to increase availability. An ASC-specific educational toolkit, which is now publically available on AHRQ's website, was designed and incorporated into standard content.

A QI framework was developed to help facility leads track and report their progress in implementing the ASC surgical safety checklist and other QI initiatives, along with an imple- 
mentation guide to provide step-by-step guidance on how to implement the checklist in the ASC environment. Though implementation was supported by partnerships between national stakeholders in all models, the addition of the EFN in the QIA model allowed participating facilities access to education led by experts to whom they may not have otherwise had access.

All health care environments, including ASCs, are challenged in collecting robust data because of a lack of fully reliable feedback systems. In addition, national estimates of infections originating in ASCs were not available at the start of this project [10]. Data submission rates, even with the improved model, still were low and varied from cohort to cohort. Regardless of implementation strategy chosen, project evaluation based solely on outcome data in the ASC environment is difficult because of the low rates of adverse events and challenges collecting data. Low data submission rates with high attrition were observed throughout the course of each cohort. While the reason for low data submission is unknown, potentially this may be because continued participation was not dependent on reporting data, or facilities were already submitting data to other organizations. Future interventions may wish to try other approaches to incentivize data submission or reduce the data submission burden on facilities.

The types of events reported provided additional insight into complications in this environment. In the future, standardizing the feedback loop to ASCs from all health care settings will be important to the collection of data regarding adverse and unexpected events. This would allow for a more accurate accounting of the breadth and frequency of events and allow for deployment of resources for improvement in to those areas. As of May 2016, the CMS Quality Reporting Program has begun publicly reporting ASC outcome measure data at the provider, state, and national levels so that facilities will be able to benchmark against a larger number of other facilities in future national quality improvement work. Future interventions could incorporate this publicly available data.

\section{Limitations}

Not all elements of this approach apply to other settings, but there are some overarching implementation principles that apply across all health care environments, including ASCS. The NPT implemented a series of rapid-cycle changes in order to grow and adapt to the needs of participating ASCs in the project. Because of this approach, changes made were not grounded in rigorous research methodology. Not all project teams will have access or resources to pull together a broad, interdisciplinary group of national organizations and experts to implement QI projects. As such, suggestions in this paper may not be applicable to all QI projects in ASCs.

\section{Conclusions}

In order for QI projects to be successful in the ASC environment, project design and implementation must be tailored to this setting. While some of the approaches that have been successfully used in inpatient hospital settings on previous QI projects work, many do not translate well and require significant modification. Using rapid-cycle analysis QI strategies in implementation ensured the fluidity and ability of the team to adapt throughout the project; however, significant project time was spent learning about the environment itself and improving the intervention. The success of this project depended on leveraging a network of national partners with a wide variety of strengths in an effective way to ensure all aspects of safe surgical care were addressed.

The NPT learned many lessons over time through a process of continuous change and iterative improvement. These lessons could be used to help others shape future work by potentially learning from the changes that were made throughout this intervention. Tailoring interventions to account for the needs of ASCs and the issues surrounding data collection in this setting will help continue QI advances in ASCs and allow project time to be spent on implementation rather than process refinement.

\section{Disclosure}

The opinions expressed in this document are those of the authors and do not reflect the official position of AHRQ or the U.S. Department of Health and Human Services.

\section{Conflict of Interest}

No authors reported any conflict of interest.

\section{Funding Support}

This project was funded under contract number HHSA2902010000251 from the Agency for Healthcare Research and Quality (AHRQ), U.S. Department of Health and Human Services. The Agency for Healthcare Research and Quality (AHRQ) funded a multi-cohort, quality improvement (QI) collaborative for the ambulatory surgery environment entitled AHRQ Safety Program for Ambulatory Surgery. HRET was tasked with the management of the project contract over a 4-year period from September 30, 2012 to September 29, 2016. The purpose of the contract was to adapt the Comprehensive Unit-based Safety Program (CUSP), which was initially developed for the acute care setting, for use in ASCs. The project was a collaborative effort with key partners, including Harvard T.H. Chan School of Public Health (HSPH), Ambulatory Surgery Center Association (ASCA), ASC Quality Collaboration (ASC QC), South Carolina Hospital Research and Education Foundation (SCHREF), Institute for Healthcare Improvement (IHI), and Westat (see: http://www.hret.org/dissemination/projects/ascambulatory-safety.shtml).

\section{References}

1. Ambulatory Surgery Center Association. History of 
ASCs. Available at: http://www.ascassociation.org/advancingsurgicalcare/whatisanasc/historyofascs. Accessed September 1, 2016.

2. Jani SR, Shapiro FE, Gabriel RA, Kordylewski H, Dutton RP, Urman RD. A Comparison between office and other ambulatory practices: Analysis from the National Anesthesia Clinical Outcomes Registry. J Healthc Risk Manag. 2016;35(4):38-47.

3. Harrison S. Leadership and compliance in the ASC environment. AORN Journal. 2016;104(1):16-17.

4. Russo A, Elixhauser A, Steiner C, Wier L. Hospital-based ambulatory surgery, 2007: statistical brief \#86. Rockville: AHRQ; 2010.

5. Fleisher LA, Pasternak LR, Herbert R, Anderson GF. Inpatient hospital admission and death after outpatient surgery in elderly patients: importance of patient and system characteristics and location of care. Arch Surg. 2004;139(1):67-72.

6. Agency for Healthcare Research \& Quality. Action II, request for task order: development and demonstration of a surgical unit-based safety program (SUSP) to reduce surgical site infections (SSI) and other surgical complications. Rockville: AHRQ; 2011.

7. Agency for Healthcare Research \& Quality. Eliminating CLABSI, a national patient safety imperative: final report. Available at: http://www.ahrq.gov/professionals/ quality-patient-safety/cusp/clabsi-final/index.html. Accessed September 1, 2016.

8. Saint S, Greene MT, Krein SL, Rogers MA, Ratz D, Fowler KE, Edson BS, et al. A Program to Prevent Catheter-Associated Urinary Tract Infection in Acute Care.
N Engl J Med. 2016;374(22):2111-2119.

9. World Health Organization. WHO guidelines for safe surgery: 2009: safe surgery saves lives. Geneva: World Health Organization. Available at: http://apps.who.int/ iris/bitstream/10665/44185/1/9789241598552_eng.pdf. Accessed September 1, 2016.

10. Agency for Healthcare Research \& Quality. Action II, request for task order: development and demonstration of a surgical unit-based safety program in ambulatory surgery (SUSP-AS) to reduce surgical site infections (SSI) and other surgical complications. Rockville: AHRQ; 2012.

11. Fakih MG, George C, Edson BS, Goeschel CA, Saint S. Implementing a national program to reduce catheter-associated urinary tract infection: a quality improvement collaboration of state hospital associations, academic medical centers, professional societies, and governmental agencies. Infect Control Hosp Epidemiol. 2013;34(10):10481054.

12. Molina G, Jiang W, Edmondson L, Gibbons L, Huang LC, Kiang MV, Haynes AB, et al. Implementation of the Surgical Safety Checklist in South Carolina Hospitals Is Associated with Improvement in Perceived Perioperative Safety. J Am Coll Surg. 2016;222(5):725-736 e725.

13. Agency for Healthcare Research \& Quality. Ambulatory surgery center survey on patient safety culture. Available at: http://www.ahrq.gov/professionals/quality-patientsafety/patientsafetyculture/asc/index.html. Accessed September 1, 2016.

14. Allison J. Ideas and Approaches for Quality-Assessment and Performance-Improvement Projects in Ambulatory Surgery Centers. AORN J. 2016;103(5):483-488. 